\title{
Exposure and Dose: Health Effect Studies Associated with Nanometer Aerosols
}

\section{Lev S. Ruzer}

Lawrence Berkeley National Laboratory, USA

\begin{abstract}
The nanotechnology industry is rapidly growing with promises of substantial benefits that will have significant global economic and scientific impacts applicable to a whole host of areas from engineering and electronics to environmental remediation and medical healthcare. However, at present there is growing concern over the safety of nanomaterials with respect to occupational, consumer and environmental exposures and associated health effect. Particularly, information on nanomaterial exposure, dosimetry, risk assessment and health effect is negligible. In this paper we discussed the problems of the Exposure and Dose in the studies of the health effect related to nanoaerosols. As a general plan for the assessment of the Dose - the main cause of the effect - Nanoparticles Dosimetric Road Map is presented. The new ideas for measurement of the surface area of nanoparticle, local nanoparticle lung deposition, and nanoparticles respirators true effectiveness are proposed.
\end{abstract}

\section{Introduction}

The nanotechnology industry is rapidly growing with promises of substantial benefits that will have significant global economic and scientific impacts applicable to a whole host of areas from engineering and electronics to environmental remediation and medical healthcare. However, at present there is growing concern over the safety of nanomaterials with respect to occupational, consumer and environmental exposures and associated health effect.

Particularly, information on nanomaterial exposure, dosimetry, risk assessment and health effect is negligible.

According to Kulinowski, et al. [1] "there's a lot more data now than there was back in the early days. Between 2001 and 2008 (the last year for which complete data are available), the annual NanoEHS publication rate grew between $20-120 \%$ per year with over 3600 individual papers".

Unfortunately, however, "it becomes equally difficult to say that all these data are conclusive. A recent analysis found that much of the "nanotoxicology" research is done in vitro, focusing on acute toxicity and mortality induced by native nanoparticles, with limited relevance to human health".

Up to present time, available quantitative data related to dosimetry of nanomaterials, and particularly aerosoloized nanoparticles are very difficult to find.

As it discussed in in terms of nanoparticles [2] we still don't know: nature and extent of hazard; nature and extent of exposure; nature and extent of risk;

What measure to use; limitation of controls; limitations of protection;

What limits are appropriate? There are no specific exposure limits;

According to [3] there is "no consistent nomenclature, terminology and measurement standards to characterize and describe nanoparticles and exposure. Inadequate understanding of nanotoxicity, in particular to determine whether acceptable exposure limits exist. No effective methods to measure and assess workplace exposure to nanoparticles; no data on existing or predicted workplace exposure.

Despite the hundreds of products containing nanomaterials that are already being manufactured commercially, and the emerging body of scientific literature demonstrating the serious risk associated with nanotoxicity, there are still no laws to manage workplace exposure and to ensure workers' safety. This suggested that governments have learnt little from their experiences with asbestos".

The health effects from aerosols in air pollution are discussed widely in the scientific literature. During the last 10 years the need to understand the relationships between aerosol exposure and biological effects has became specially important due to rapid development of new, revolutionary industry - nanotechnology.

Understanding of the relationship between aerosol concentration in the breathing air and particle deposition in different portions of the lung remains poor, in spite of several decades of research.

There are many gaps in our knowledge of aerosols in the nanometer range, which we need to fill in order to improve risk assessment and dosimetry of nanoaerosols [4], including:

- Nanoparticle dosimetry and risk assessment.

- Evaluation of pulmonary deposition and translocation of inhaled nanomaterials.

- Lack in the systematic approach for the assessment of the main cause of the health effect - nanoaerosol dose.

- Measurement studies of nanoparticle surface area concentrations in the workplace.

Some of the gaps are substantial for aerosols in all ranges of sizes, but majority are especially important mostly for the particle in the range $1-100 \mathrm{~nm}$.

Corresponding author: Lev S. Ruzer, Lawrence Berkeley National Laboratory, USA, E-mail: Iruzer@aol.com

Received September 27, 2011; Accepted November 21, 2011; Published November 23, 2011

Citation: Ruzer LS (2011) Exposure and Dose: Health Effect Studies Associated with Nanometer Aerosols. J Nanomedic Nanotechnol 2:120. doi:10.4172/21577439.1000120

Copyright: (c) 2011 Ruzer LS. This is an open-access article distributed under the terms of the Creative Commons Attribution License, which permits unrestricted use, distribution, and reproduction in any medium, provided the original author and source are credited. 
The problem presented in this paper in the form of nanoaerosol dosimetric road map, where the nanoaerosol dose assessment is divided on particular steps each of it contributed to the main dosimetric goal.

According to EPA USA, [5] "In epidemiological studies, an index of exposure from personal or stationary monitors of selected pollutants is analyzed for associations with health outcomes, such as morbidity or mortality.

However, it is a basic tenet of toxicology that the dose delivered to the target site, not the external exposure, is the proximal cause of a response.

Therefore, there is increased emphasis on understanding the exposure-dose-response relationship.

Exposure is what gets measured in the typical study and what gets regulated; dose is the causative factor. The measurement or assessment of the dose in practice, however, present substantial difficulties, and as a surrogate of the dose the term Exposure is used.

\section{Exposure definitions}

The term "exposure" came to the aerosol field from the study of the effect of gases.

Aerosol science, including epidemiological studies of the health effect of aerosol exposure, was developed mainly in the second half of the 20th century. In 1924, German chemist Fritz Haber [6] proposed the following definition of exposure:

For each war gas, the amount (c) present in one cubic meter of air expressed in milligrams and multiplied by the time $(\mathrm{t})$ in minutes necessary for the experimental animal inhaling this air to obtain a lethal effect. The bigger this products $\left(c^{\star} t\right)$, the greater is the toxicity of the war gas.

The definition of exposure in the case of aerosols is much more complicated. The main difference in dosimetry between gases and aerosols is that aerosol distribution spatially and temporally uneven and deposited aerosol in the lungs is nonuniform. The reason for this is that respirable fractions of aerosols consist of particles with diameters ranging from nanometers to $5-10 \mu \mathrm{m}$. Therefore, their airborne particle size distribution and their deposition inside the lungs are very uneven.

According to the National Academy of Science report [7], the definition of exposure is:

"An event that occurs when there is contact at a boundary between humans and the environment with a contaminant of a specific concentration for an interval of time; the units are concentration multiplied by time".

From our point of view this definition is not free from some contradiction. If exposure is an event, so it cannot be expressed in physical units.

A statistical definition of exposure has been proposed [8]: "An exposure at some instant of time is a joint occurrence of two events: 1. the pollutant of concentration $\mathrm{C}$ is present at a particular location in space at a particular time, and 2. the person is present at the same time and location in space."

A later definition [9] addresses the notion that the target remains important, and also that different parts of the target can receive different exposures at the same time.

The last definition is more adequate for aerosols, because it takes into account the specific aerosol problem of non-uniformity.
In the Guidelines for Exposure Assessment [10] a slightly different definition was proposed:

Exposure - Contact of a chemical, physical, or biological agent with outer boundary of an organism. Exposure is quantified as the concentration of the agent in the medium in contact integrated over the time duration of that contact.

In all these definitions, the key word is contact, which means that in the case of aerosols only breathing zone measurement concentration or particle size distribution should be used for the exposure and particle size measurement. If concentration (and particle size characterization) is provided at a distance from the breathing zone, the correlation should be established between breathing zone and sampling site measurement.

The problem with all these definitions that it is not clear in which of them the exposure is a physical value or an event. But is it clear from all exposure definitions this term should not be used for the substitution of the dose and as a cause of the effect.

In [11] we present the definition of dose in case of aerosols:

Dose is the specific quantity of aerosols delivered to a target site that is directly responsible for a biological effect

\section{The term "quantity" is defined as follows:}

1. In the case of radioactive aerosols, deposited energy per unit mass for alpha, beta, or gamma radiation is expressed in units of $\mathrm{J} / \mathrm{kg}$ (Gray) or rads $(100 \mathrm{erg} / \mathrm{g})$ or the equivalent.

2. In case of nonradioactive aerosols, quantity is the deposited number of particles, surface area, or mass of a discrete particle size.

3. The term "directly" means that dose is a quantity of the deposited amount of aerosol particles after the completion of all biokinetic processes.

Unfortunately, the term dose is easier to define than measure in practice. Therefore in many studies the Exposure is measured as a concentration multiplied by time, and attempt were made to find correlation between Exposure (which is, strictly speaking, only surrogate of the cause of effect - dose) and the health effect. We present two of such studies.

\section{Examples of Exposure-effect Study Without Dose Assessment}

I think it make sense to analyze these studies especially because we are now at the beginning of the new global problem - assessment of the dose and health effect of nanoparticles.

The similarity is that in both case we have to study two groups of separate problems - dosimetry and a health effect.

The difference is that in case of radiation in general and problem of radon and its progeny in particular there were many studies on correlations between radon and its progeny both in terms measurements and health effect, including on human. Unfortunately, similar data for nanoparticles are not available right now.

I will try to illustrate this with results of two study: [12], and Darby, et al. [13].

It should be mentioned that in BEIR VI a great work was accomplished - the distribution of radon and its progeny concentrations in mines of 11 different countries were presented and analyzed together with data on lung cancer mortality among miners.

In Darby, et al., the similar very important data on radon 
concentrations in different countries in Europe were collected and analyzed. Again, the authors did a very good job in collecting and analyzing information on distribution of radon concentration in Europe.

Unfortunately they try to connect radon concentration itself with lung cancer mortality of general population, despite the fact that only the radon progeny, not radon itself, are responsible for the biological effect. So, it is classic case when not a dose as a cause of the effect, but rather surrogate for dose, and the correlation between and real cause was not established. But that is not all.

In this study the authors found that "the absolute risks of lung cancer by age 75 years at usual radon concentrations of 0,100 ,and 400 $\mathrm{Bq} / \mathrm{m}^{3}$ would be about $0.4 \%, 0.5 \%$, and $0.7 \%$, respectively, for lifelong non-smokers, and about 25 times greater $(10 \%, 12 \%$, and $16 \%)$ for cigarette smokers".

It is obvious that if we take into account uncertainty in the risk assessment in this case the numbers $(0.4 \%, 0.5 \%$, and $0.7 \%)$ and $(10 \%$, $12 \%$, and $16 \%$ ) will be the in the range of errors, i.e. the same.. By the way, these concentrations are considerably lower than permissible concentrations for mines.

In Table D-12 of BEIR VI results of "average exposures" in "average Working Levels" (WLM) are presented for 11 countries (China - 286.0, Czechoslovakia - 196.8, France - 59.4, Canada, USA - 578.6, etc.). We know that the uncertainty in the exposure assessment is in the order at least of tens of percents. So, we cannot trust these data with tenths of WLMs.

Our study on dosimetry and health effect of miners in Tajikistan (Ruzer, et al 1995, 11] suggested that different groups of miners got substantially different exposure (dose) and different lung cancer mortality. So averaging in this case can lead to additional uncertainty.

It seems, that in epidemiological studies exist some sort of tendency to present as much as possible cases of effect (mortality, morbidity, etc.) in order to get good statistic. With such tendency we often used old and questionable data on concentrations, even based on data on ventilation. So, our good statistic on mortality in such case, is compromise with bad dosimetry.

\section{Nanoparticle Definition}

In aerosol science, a particle is defined as a small object that behaves as a whole unit in terms of its transport and properties. It is further classified according to size: In terms of diameter, fine particles cover a range between 100 and 2500 nanometers, while ultrafine particles, on the other hand, are sized between 1 and 100 nanometers. Similarly to ultrafine particles, nanoparticles are sized between 1 and 100 nanometers, though the size limitation can be restricted to two dimensions.

Nanoparticles may or may not exhibit size-related properties that differ it significantly from those observed in fine particles or bulk materials.

There is no accepted international definition of a nanoparticle, but one given in the new PAS71 (the British Standards Institution, BSI) document developed in the UK is: "particle with one or more dimensions at the nanoscale". Correspondingly, the nanoscale is defined as "having one or more dimensions of order of $100 \mathrm{~nm}$ or less". There is a note associated with this definition: "Novel properties that differentiate nanoparticles from the bulk material typically develop at a critical length scale of under $100 \mathrm{~nm}$ ".

This makes the size of particles or the scale of its features the most important attribute of nanoparticles.

\section{What is different about a nanoparticle?}

There is no strict dividing line between nanoparticles and nonnanoparticles.
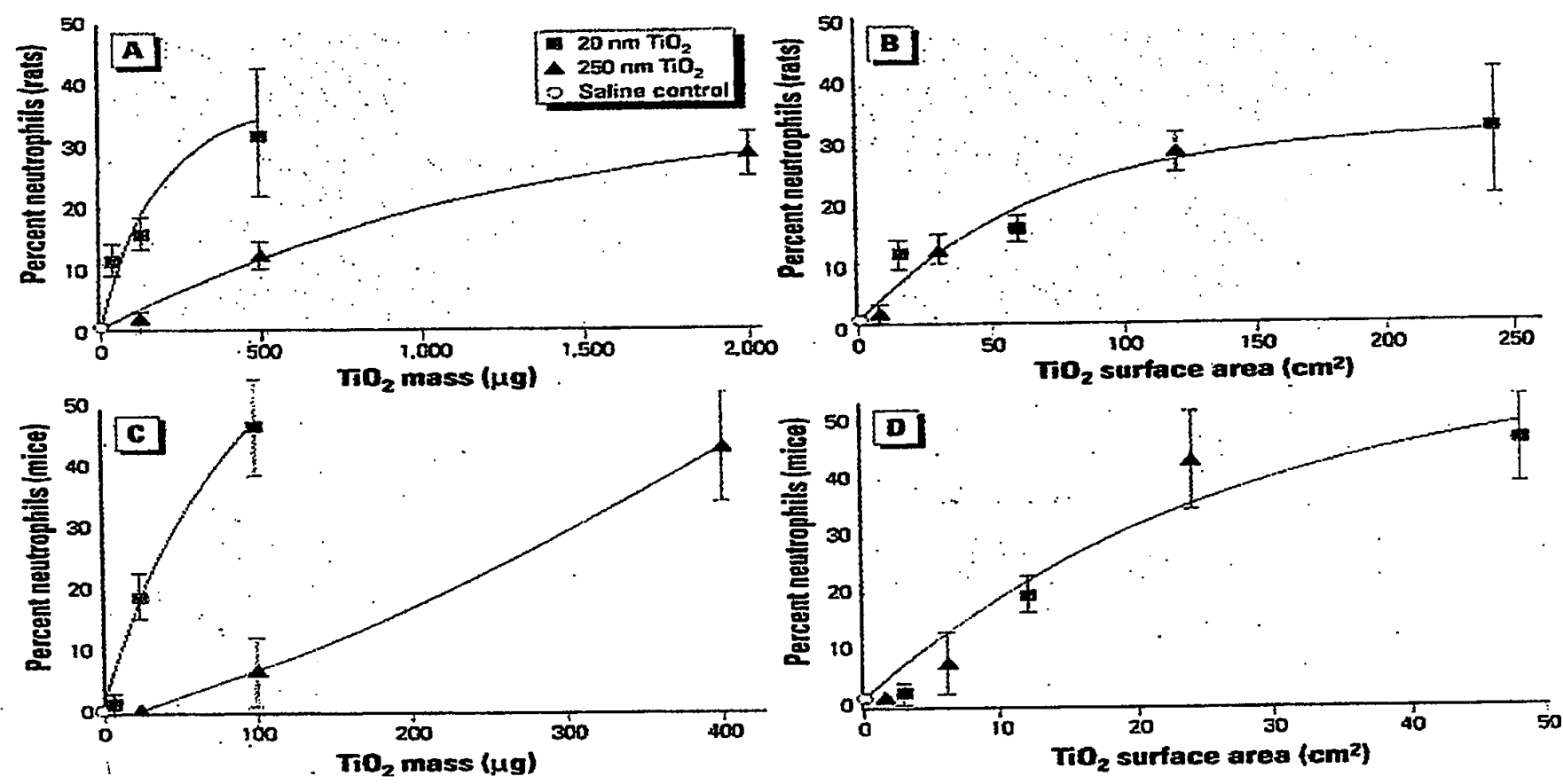

Figure 1: Effect vs aerosol mass, surface area concentration Oberdörster $\mathrm{G}$ et al. [14]. 
The size at which materials display different properties to the bulk material is material dependant and can certainly be claimed for many materials much larger in size than $100 \mathrm{~nm}$. Most experts in UK and USA define nanoparticles as particles smaller than $100 \mathrm{~nm}$. But, for example in Japan, particles between 50 and 100 $\mathrm{nm}$ are classified as "ultrafine" and only those below $50 \mathrm{~nm}$ in one dimension as nanoparticles. Even some agencies in USA use the term "ultrafine" to describe particles under $100 \mathrm{~nm}$ (usually in connection with natural or incidental nanoparticles).

Data on Figure 1 demonstrated that nanoparticles appeared to be more inflammatory in lung than microscale particles. But if the data were plotted against surface area instead of mass, response was identical for both nano and larger particles [14]. In other words in principle there is no difference between the nanoparticles, fine particles and bulk materials. The difference i that in case of nanoparticles the ratio of number of molecules on the surface to all molecules of particle "surface to volume ratio", (SVR) is much higher than that of fine particles or bulk materials.

The graph illustrating the portion of molecules on the surface to all molecules of the particle as a function of particle size is shown on Figure 2.

It is clear from this graph that for nanoparticles close to $1 \mathrm{~nm}$ in diameter the surface area to volume ratio is close to $1(100 \%)$. In this paper we proposed to use radioactive particles with the size of $1 \mathrm{~nm}$ in diameter and diffusion coefficient $\sim 0.06 \mathrm{~cm}^{2} \mathrm{~s}^{-1}$ as an experimental tool in the study of nanoparticles.

It is also clear from the same graph that for spherical particles with diameter of $100 \mathrm{~nm}$ the surface to volume ratio will be $\sim 0.03$ (3\%). So the range of sizes of nanoparticle in terms of surface area to volume is from 0.03 to 1.0 .

Based on this consideration we propose another definition for nanoparticles based on the portion of molecules on the surface to all molecules of the particle. As an appropriate number for this boundary portion we propose $3 \%$ or 0.03 , which according to graph Figure 2 correspond to spherical particles with diameter of $100 \mathrm{~nm}$.

"Nanoparticles are particles which have the ratio of number of molecules on the surface to all particle molecules - surface to volume ratio (SVR) of $3 \%(0.03)$ or higher".

\section{Nanoparticle Dosimetric Road Map}

Figure 3 presents a conceptual mapping of the processes starting with the manufacture of nanomaterial, through its possible release into the environment and ultimate dose to the target cell in lungs and other organs.Let's consider every step in our Dosimetric Nanoaerosols Road Map from the point of view of available approach, methods and measurement technique. We begin with the left branch which describes the study of airborne nanoparticles.

Manufacturing and handling processes for nano-sized materials are widely variable. For example the materials may be fabricated in a fluidic system closed to the environment (e.g., colloidal suspension of metallic crystals formed in a liquid reaction vessel), or in an open-air system where they may directly mix with ambient air (e.g., manufacture of carbon black using combustion techniques). For each anotechnology the potential for fugitive emissions leading to an airborne concentration can be different. Information on the release of the nanomaterial to the air for different nano-material manufacturing is very scarce.

In this paper we will discuss some new ideas and experimental data on the assessment of parameters presented in Nanoparticles Dosimetric Road Map.

It should be notice that the problem of metric in nanoaerosol concentration is complicated. First, nanoaerosols often exists in practice as a structure not as a single particle with the size at least in one dimention in the range from 1 to 100 in diameter. So, generally speaking, it is not always possible to use diameter itself as a characteristic of the particle.

Second, from the point of view of the dose to the lung the aerosol mass concentrations as a characteristic also have many disadvantages:

1. In the nanometer range when mass concentration is very small, the number concentration, particle density at the lung tissue, and correspondingly dose can be very high.

2. With the same mass concentration particle size distribution can be different, so particle deposition inside the lung will differ along with the correspondingly dose and biological effect.

3. In case of aerosols for the dose assessment only the "respirable" particles, i.e. particles with diameter less than 5-7 $\mu \mathrm{m}$ are important. Unfortunately, this limit is itself uncertain [15]

According to the majority of the studies of ultrafine and nanosized aerosols, it is not the mass oncentration, but particle number and surface area concentrations that should be used for the assessment of dose because they appear to be better predictors of health effects $[14,16,17]$.

In this paper we present the new idea on nanoparticle surface area measurement.

\section{Nanoparticles surface area measurements}

Particle surface area is currently considered to be an important toxicological criterion for assessing inhalation exposure. Currently, however, there is a need to develop and expand mehods available by which particle surface area concentrations can be assessed in the workplace. The main concern is with free nanoaerosols that are more

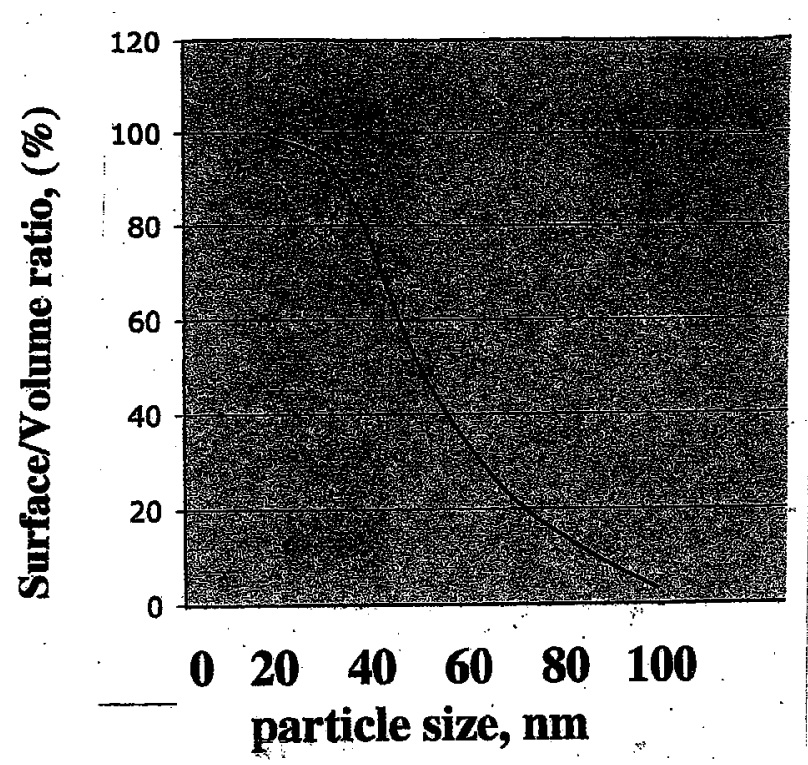

Figure 2: Nanoparticles surface / volume ratio (\%) vs particle size, nanometers. 


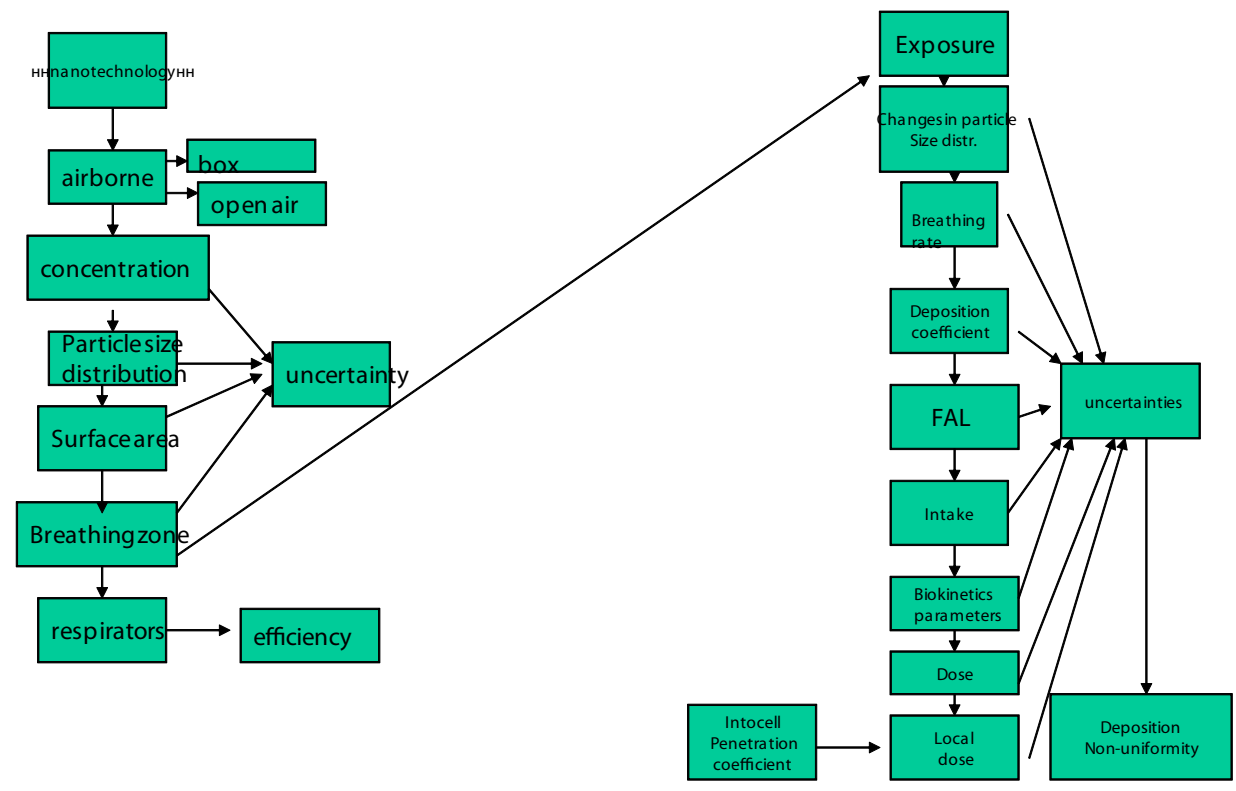

Figure 3: Nanoparticles dosimetric road map.

available for absorption and distribution within the body. Investigators found that when lung burdens and clearance rates were expressed as a function of the surface area, there was a much closer correlation with biological responses [18].

The special importance of the surface area of nanoparticles in the study of the risk assessment is discussed in [19] by comparing the three characteristics: particle number, surface area, and mass concentration.

The use of particle surface area as a dose metric for nanoaerosols is discussed in many reports: NIOSH [20,21,22], Royal Society (2004), Aitken et al. [17], DEFRA [23], SCENIHR [24], Renn and Roco [25], and ASCC [18]

Our proposed approach [25] represents a synthesis of:

1. Derived direct analytical correlation between the "unattached fraction" of radon progeny and surface area particle concentration in the range of 1-100 $\mathrm{nm}$ particle diameter;

2. Experimental data on correlation between the unattached fraction of radon progeny and particle surface area for particle diameter in the range of $44 \mathrm{~nm}-2.1 \mu \mathrm{m}$.

One important property of radon decay products is that after alpha decay of radon, the newly formed atom of ${ }^{218} \mathrm{Po}$ becomes surrounded by small number of molecules (around 10 to 12). Such molecular clusters, referred to as unattached progeny, with sizes in the range 1-4 $\mathrm{nm}$ and diffusion coefficients of approximately $0.06 \mathrm{~cm}^{2} \mathrm{~s}^{-1}$, can be potentially useful as a marker in the studies of properties of nonradioactive aerosols, especially nanoaerosols. Figure 4 depicts the basic processes of gaseous radon decay producing unattached progeny. These progeny may remain unattached, or may become associated with existing aerosols. It seems reasonable that there should be some correlation between unattached activity and aerosol concentration. It is likely that lower aerosol concentrations will lead to larger fractions of unattached activity. If this relationship can be quantified, then the unattached activity fraction of radon progeny can be used as a measure of airborne particle concentration.
1. This idea [26] was illustrated by an experimental study with spherical polystyrene particles in the size range 0.3-2.0 $\mu \mathrm{m}$, i.e. outside of the nanometer range [27]. Figure 5 shows experimental results of correlation between the unattached fraction of radon decay products and the aerosol surface area measured in these experiments. The methods for measuring the unattached fraction of radon decay products and all of the necessary corrections are described in [28]. The procedure for measuring the relationship between the unattached activity fraction and particle concentration consists of passing the radon-exposed air through a device with two channels: one with only an open filter, and one with a diffusion battery or other

The measurement of these parameters should be provided by means of an "unattached activity generator", a device capable of generating a regulated unattached activity fraction. Such generators were described in Dokukina and Ruzer [27] and Butterweck et al. [29].

\section{Correlation between nanoparticles surface area and unattached activity}

Deposition processes for unattached radon decay products to aerosol particles has been studied both theoretically and experimentally $[30,31]$. This work showed that for particles smaller than $10 \mathrm{~nm}$, the probability of deposition attachment is proportional to the square of the particle radius, and for particles larger than $1 \mu \mathrm{m}$, deposition is proportional to the particle radius. Those studies determined the general formula for the constant of deposition, $\lambda_{\text {at }}$, of the unattached activity to aerosol particles in equilibrium to be:

$$
\lambda_{\alpha \tau}=(1 / \tau)=4 \pi \rho^{2} \mathrm{~N} \beta /(1+\eta \rho)=\Sigma \beta /(1+\rho \eta)
$$

where $\tau$ is the time for the concentration of unattached activity to decrease by a factor of $1 / \mathrm{e}, \mathrm{r}$ is the particle radius, $\mathrm{N}$ is the particle number concentration, $\beta=(\mathrm{v} / 4) \sim 4300 \mathrm{~cm} \mathrm{~s}^{-1}, \mathrm{~h}=\mathrm{v} / 4 \pi \mathrm{D} \sim 70000$ $\mathrm{cm}^{-1}$ and $\mathrm{S}$ is the particle surface area concentration. Here, $\mathrm{v}$ is the root-mean-square velocity of radon decay product molecules under standard conditions and $\mathrm{D}$ is the Brownian diffusion coefficient of the unattached particles. 
Croft and Perry [32] described the formation of the unattached activity from radon and its deposition onto aerosol particles and surfaces. They developed the following expression for the unattached activity fraction, $\mathrm{f}$

$$
\mathrm{f}=\lambda_{\mathrm{A}} /\left(\lambda_{\mathrm{A}}+\lambda_{\mathrm{at}}\right)
$$

where $\lambda_{A}$ is the decay constant of ${ }^{218}$ Po the first daughter product of radon decay. Combining equations (2) and (3) yields equation (4), an expression that directly correlates aerosol surface area, $\mathrm{S}$, to the unattached activity fraction $\mathrm{f}$ :

$$
S=\left(\lambda_{\mathrm{A}} / \beta\right)[(1 / \mathrm{f})-1](1+\mathrm{rh})
$$

For particles in the size range $1-100 \mathrm{~nm}$, the term $(1+\mathrm{rh})$ is approximately equal to 1 , and equation (4) can be simplified to:

$$
\left.\mathrm{S}=\lambda_{\mathrm{A}} / \beta\right)[(1 / \mathrm{f})-1]
$$

Results of calculation for ${ }^{218} \mathrm{Po}$ according to equation (5) was showed the relationship of the particle surface area concentration versus particle size for several different values of the unattached activity fraction, $\mathrm{f}$, is presented in Figure 6. For ${ }^{218} \mathrm{Po}$,

$\lambda_{\mathrm{A}}=3.788^{\star} 10^{-3} \mathrm{~s}^{-1}$

$\mathrm{S}=8.84^{\star} 10^{-7}[(1 / \mathrm{f})-1]\left(1+7^{\star} 10^{4} \mathrm{r}\right) \mathrm{nm}^{2} / \mathrm{cm}^{3}$;

Figure 6 suggests that for the same concentration of the unattached activity fraction, the surface area of the particles will not depend on particle size for particles smaller than $100 \mathrm{~nm}$ (Ruzer, Apte, 2005). Calibration of this technique should be done with monodispersed spheric particles. Practical measurements of polydispersed and nonspherical particles in the nanometer range will need to be presented in the units of "equivalent surface area", i.e., the surface area of monodispersed particles, which correspond to the same concentration of the unattached fraction.

Figure 7 present calculation of $\mathrm{S}$ vs $\mathrm{f}$ according to formla (5).

As we already mentioned the use of mass concentration data alone is insufficient for the expression of dose, and the number concentration and / or surface area need to be included.

Unfortunately there is a lack of information on measurement of nanoaerosols particles, and especially the size distribution and surface area concentration in the working environment.

Review of literature on environmental health in the new rapidly developing nanotechnology industry shows that problem of exposure has not been adequately assessed [14]. A gap exists between existing particle measurement methods and those truly appropriate for nanoaerosol exposure assessment. Until now, the primary tools available for measurement of nano-sized aerosols have been Condensation Particle Counters (CPCs), and Differential Mobility Analyzers (DMA).

A new instrument on the market, the Nanoparticle Surface Area Monitor (TSI 3550), is used for assessment of deposited surface area (DSA) in the lung. Lung deposition estimates from this instrument are based on correlations developed [33] between the electrical signal and modeled DSA. The instrument is said to be capable of detecting particles with diameters down to $10 \mathrm{~nm}$.

One of the important problem in the safety of people working with nanomaterials is the effectiveness of respirators, discussed in many reports: (Strategic Plan for NIOSH, etc)

\section{Nanoparticles respirators true effectiveness measurements}

The problem of respirator efficiency discussed in many reports:
(Nanotechnology; 2005), [17], (White Paper on Nanotechnology; (IRGC). Orwin Renn and Mike Roco Geneva, Switzerland, Shaffer). It is well known that the determining factor which governs the effectiveness of respiratory protective equipment (RPE) is not absolute penetration through the filter, but rather face-seal leakage, which bypasses the device.

In Ruzer, et al, the new idea for measuring the true efficiency of respirators was used based on direct measurement of the gammaactivity of radon progeny in lungs of human.

Let us denote

$A_{0}$ - measured gamma-activity in the lung of miners before entering radon atmosphere; (background measurement).

$A_{1}-A_{0}$ measured gamma-activity in the lung of miners without wearing respirator;

A $-A_{0}$ measured gamma-activity in the lung of miners with respirator;

Then respirator effectiveness will be $\left(A-A_{0}\right) /\left(A_{1}-A_{0}\right) 100 \%$; and

Penetration coefficient $\left\{1-\left[\left(\mathrm{A}-\mathrm{A}_{0}\right) /\left(\mathrm{A}_{1}-\mathrm{A}_{0}\right)\right]\right\} 100 \%$;

Our measurements in mines demonstrated that true respirator efficiency varies from 67 to $95 \%$ depending on individual training, duration, and type of work.

At the present time the data on the true effectiveness of respirators, including the face-leakage problem, are unavailable.

\section{Local lung deposition and dosimetry for nanoparticles}

There is a lack of systematic studies on nanoparticle lung deposition.

One of the most important difficulties in the assessment of the dose as a main cause of the effect from aerosols, particularly in the nanometer range, is the lack of information on local deposition in human lung.

Our approach to this fundamental problem we presented in (Ruzer, Apte, 2010).

Operationally, the concept uses these ${ }^{218}$ Po radon progeny as a radiolabel. These particles have a very high diffusion coefficient and readily attach to other particles in air. When attached, or aggregated, with the environmental aerosol, these particles are called "attached activity." Given their high diffusion coefficient, their attachment efficiency can approach $100 \%$. Thus almost every particle in the environmental aerosol becomes labeled with a radioactive radon progeny particle, destined to decay and emit Gamma particles $\left({ }^{214} \mathrm{~Pb}\right.$

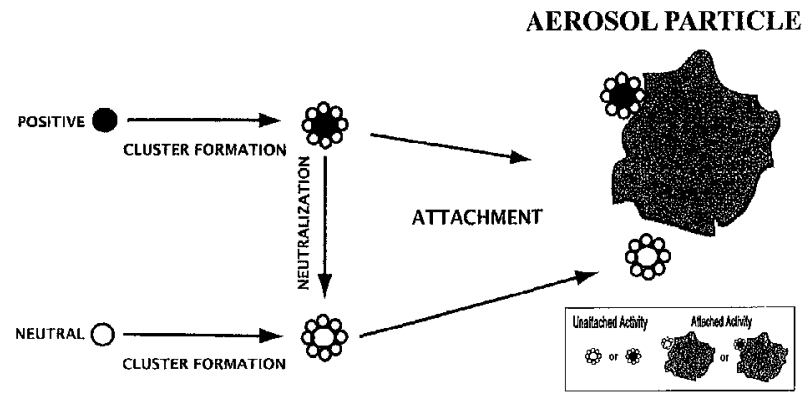

Figure 4: Basic processes of Rn decay behavior in air, defining "unattached" and "aerosol attached" activities. Clusters that do not attach to an aerosol particle are called "unattached fraction". 
and ${ }^{214} \mathrm{Bi}$ ). Particle inhalation experiments may be designed in which relatively low concentrations of radon gas is mixed with a non-active study aerosol that will subsequently be inhaled by subjects. As the radon atoms decay, their progeny attach to the study aerosol particles and thus radiolabel them.

In this case every measured gamma-quantum corresponds to a non-radioactive aerosol particle in the nanometer range locally deposited in the lung. So, the measured gamma-activity will represent dose of non-radioactive nanoaerosols at the target.

\section{Human Experiments Safety Problems}

As with all such radiotracer studies, the protocol must meet the approval of an insituttional review board (IRB) and radiological screening review. In these experiments, as in other studies, when radiation is used as a tool, for example, in using radiolabeled drugs in the study of Alzheimer disease, we have to compare the risk relative to benefit. The use of such experiments will enable us to close the gaps in our knowledge. Quantitative assessment of the local deposition of aerosol is at the core of aerosol, and particularly nanoaerosol exposure and risk assessment. So, our goal will be to find the safest possible and most appropriate marker.

The use of unattached activity of radon progeny as a radioactive tracer of deposition in the lung has many advantages:

1. Radon and its progeny belong to the natural background of radioactivity to which the general population is exposed during their lifetime. Therefore, it is easy to assess the additional risks due to its use by the methods proposed.

2. Part of radon progeny, called unattached activity, are 1-nm-sized particles with diffusion coefficient close to $0.06 \mathrm{~cm}^{2} \mathrm{~s}^{-1}$ (a size that attaches readily to nanoaerosols), which makes it very attractive as a marker for nanoaerosols with a built-in signal.

3. Radon decay products are easy to generate.

4. Radon decay products are short-lived nuclei.

This is consistent with the use of radiological tracers for other medical research. For human experiments we propose using a generator of unattached fraction of radon progeny. This could be an environmental chamber such as used in the Swiss research [29], or using a respirator mask exposure apparatus attached to a small chamber.

Human studies of exposure to radon progeny can be used to inform an assessment of the safety of its use for measurements of deposition and dosimetry of nanoparticles. If radiation exposure to a radioactive marker is negligible relative to subjects' background exposures, it may be assumed that the increased risk to the subject is negligible relative to the background exposures of subjects. The following are three documented settings of exposure to radon and its decay products:

1. Exposure in a general population with average background radon concentration of $20-80 \mathrm{~Bq} / \mathrm{m}^{3}$; and lifetime exposure duration of 600,000 hours (70 years).

2. Exposure of miners, with the permissible concentration $1100 \mathrm{~Bq} /$ $\mathrm{m}^{3}$; and exposure duration of 60,000 hours (30 work-years; [11]

3. The human exposure experiment in Paul Scherer Institute (PSI), Switzerland [29]), of $20,000 \mathrm{~Bq} / \mathrm{m}^{3}$ and duration of exposure 0.5 hours.

A comparison of these three cases shows that radiation exposure in the human experiment was less than $1 / 1000$ the magnitude of the lifetime background exposure. From a radiation exposure point of view the experiment in PSI, Switzerland, was safe.

So, will be safe experiments in the assessment of the effectiveness of respirators and local deposition of nanoparticles in human lungs in laboratory conditions presented in this paper.

\section{Conclusion}

In this paper we discussed the problem of Exposure and Dose as a measure and cause of the health effect studies associated with nanoaerosols.

In two studies of the health effect associated with radioactive aerosols presented in this paper where not the dose itself but rather surrogates of the dose - exposure - were used was demonstrated that it is impossible to make conclusive assessment of the risk associated with radon and its decay products. It make sense to analyze these studies especially because we are now at the beginning of the new global problem - assessment of the dose and health effect of nanoparticles.

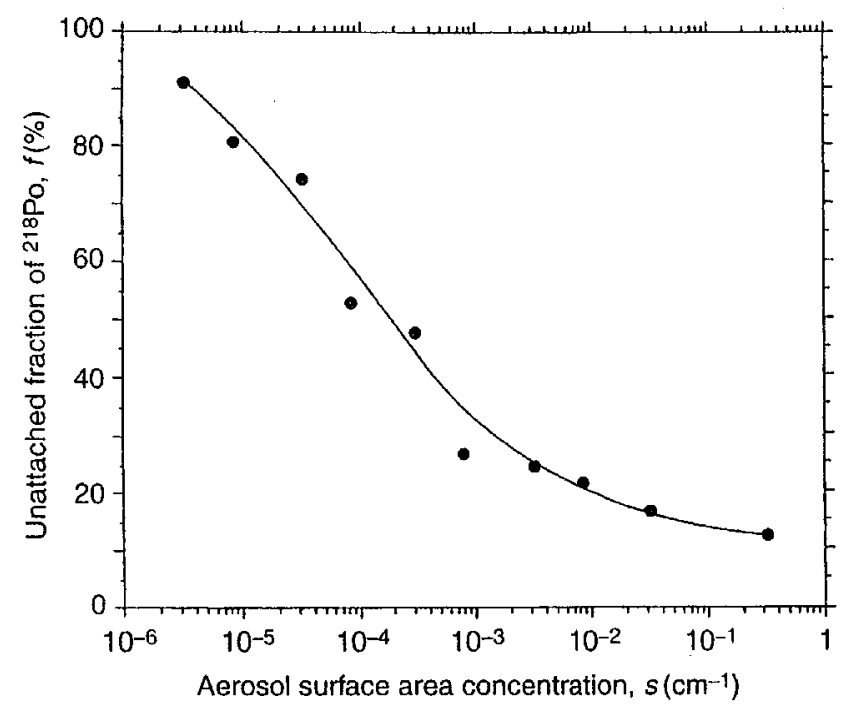

Figure 5: Unattached fraction vs particles surface area (experiment, Dokukina, Ruzer, 1976)

Correlation Between Aerosol Surface Area and Unattached Radium A $\left({ }^{218} \mathrm{Po}\right)$

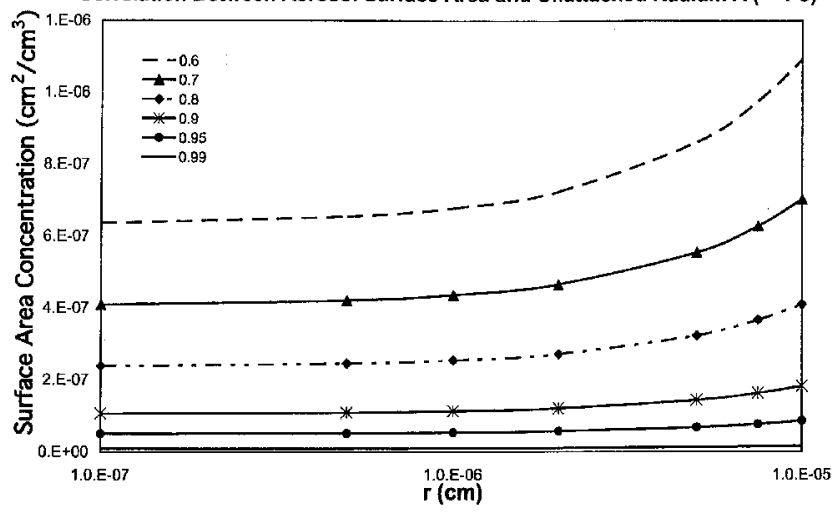

Figure 6: Relationship between particle surface area and particle radius (theory, Ruzer, 2008 [25]). 


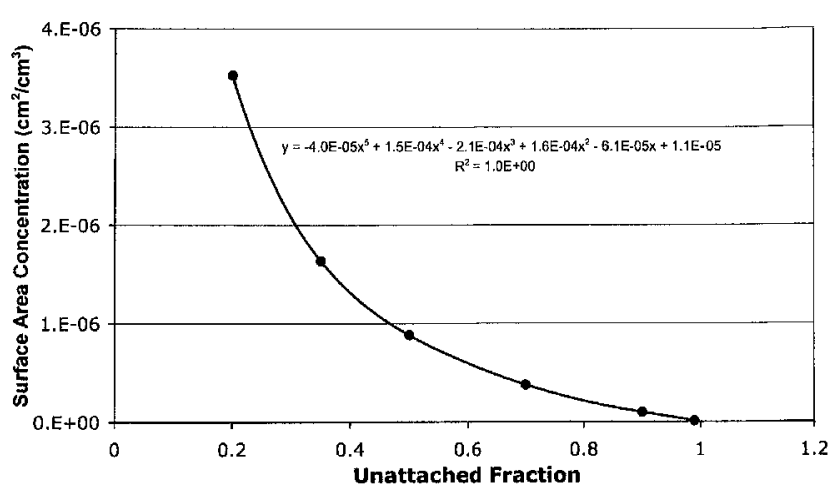

Figure 7: Theory, Ruzer, 2008 [25]

The similarity is that in both case we have to study two groups of separate problems - dosimetry and a health effect.

The difference is that in case of radiation in general and problem of radon and its progeny in particular there were many studies on correlations between radon and its progeny both in terms measurements and health effect, including on human. Unfortunately, similar data for nanoparticles are not available right now.

From presenting in this paper different definitions of the exposure it is clear that some of definition are not free from contradictions, because in some cases exposure considered as a physical value in units of concentration multiplied by time, and others as an event.

It is very important that exposure cannot be considered as a cause of the effect and strictly speaking cannot be used as a substitute of the dose. Of course we understand that in practice the measurement of the exposure is more simple and cheaper than the dose assessment.

We proposed in this paper a new definition for nanoparticle based on ratio of molecules on the surface of particle to all molecules (surface to volume ratio, $\mathrm{S} / \mathrm{V})$.

We suggest that in the study of the health effect associated with nanoparticles we have to assess the dose not an exposure as a measure of the effect.

Therefore we propose Nanoparticles Dosimetric Road Map (NDRM), consisted of two main branches: assessment of the airborne nanoparticles and assessment of local depositon of nanoparticles in the lung. (NDRM) presents a conceptual mapping of the processes starting with the manufacture of nanomaterial, through its possible release into the environment and ultimate dose to the target cell in lungs and other organs.

We presented a new idea for measuring the surface area of nanoparticle by using $1 \mathrm{~nm}$ radioactive marker unattached fraction of radon progeny.

The same tool we proposed for the safe assessment of the local deposition of nanoparticles in the lung of human and measurement of true effectiveness of respirators for naoparticles in laboratory conditions.

\section{References}

1. Kristen M. Kulinowski, Director, international council on Nanotechnology; Executive Director, Center for Biological and Environmental Nanotechnology Temptation, Temptation: Why Easy Answers About Nanomaterial Risk are probably wrong.
2. Geraci, Charles L (2009) An Update of NIOSH Nanotechnology Research Program, UC, School of Public Health. Nanoparticle Safety Symposium.

3. Friends of the Earth Nanotechnology Project (2009) AzoNano.com; Workplace Exposure to Nanomaterials and the Question of Will Nano Be the Next Asbestos.

4. Duan N, Ott WR (1990) Proceedings of the EPA/A\&WMA Conference, Las Vegas, NV.

5. EPA (1996) Review of the National Ambient Air Quality Standards for Particular Matter: Policy Assessment of Scientific and Technical Information, OAQPS Staff Paper, EPA/452/R-96-013, Office of Air Quality Planning and Standards Research Triangle Park, NC.

6. Hansspeter Witschi (1999) Some notes on the History of Haber's Law, Toxicological Sciences 50, 164-168.

7. Martonen TB, Katz I, Fults K, Hickey AJ (1992) Use of analytically defined estimates of aerosol respirable fraction to predict lung deposition patterns. Pharm Res 9: 1634-1639.

8. Ott WR (1966) Concepts of Human Exposure to Air Pollution. Environ Int 7 179-196.

9. NAS (1991) Human Exposure Assessment for Airborne Pollutants, Nationa Research Council, Washington, DC.

10. Zartarian V, Bahadori T, Mckone T (2005) adoption of an official ISEA glossary. J Expo Anal Environ Epidemiol 15: 1-5.

11. Ruzer LS, Naomi H Harley (2004) Aerosol Handbook: Measurement Dosimetry, and Health Effects, New York, CRC Press.

12. Health Effect of Exposure to Radon (1999) BEIR VI, National Research Council, Washington, DC, National Academy Press.

13. Darby S, Hill D, Auvinen A, Barros-Dios JM, Baysson H, et al. (2004) Radon in homes and risk of lung cancer: collaborative analysis of individual data from 13 European case-control studies.

14. Oberdörster G, Oberdörster E, Oberdörster J (2005) Nanotoxicology: An Emerging Discipline Evolving from Studies of Ultrafine Particles. Environ Health Perspect 113: 823-839.

15. Martonen TB, Katz I, Fults K, Hickey AJ (1992) Use of analytically defined estimates of aerosol respirable fraction to predict lung deposition patterns. Pharm Res 9: 1634-1639.

16. Hankin, CL Tran, B Ross, K Donaldson, V Stone, Chaud Q ( 2008) IOM Project CB0407, CELL PEN : A study to identify the physico-chemical factors controlling the capacity of nanoparticles to penetrtate cells.

17. Aitken RJ, Creely KS, Tran CL (2004) Nanoparticles: An occupational hygiene review. Prepared by the Institute of Occupational Medicine for Health and Safety Executive, Edinburgh; Research Report 174.

18. ASCC (2006) A Review of the Potential Occupational Health and Safety Implications of Nanotechnology. Australian Governement; Australian Safety and Compensation Council.

19. Maynard AD (2003) Estimating aerosol surface area from number and mass concentration measurements. Ann Occup Hyg 47:123-144.

20. NIOSH (2005) Strategic Plan for NIOSH Nanotechnology Research-Filling the Knowledge Gaps. Atlanta: Nanotechnology Research Program, National Institute for Occupational Safety and Health, Centers for Disease Control and Prevention.

21. NIOSH (2006a) Approach to Safe Nanotechnology.

22. NIOSH (2006b) Current Ultrafine Particle and Nanotechnology-Related Research. Atlanta: National Institute for Occupational Safety and Health Centers for Disease Control and Prevention.

23. DEFRA (2007) Characterising the Potential Risks posed by Engineered Nanoparticles; A Second UK Government Research Report. London: Department for Environment, Food and Rural Affairs.

24. Renn O, Roco M (2006) Nanotechnology Risk Governance, International Risk Governance Council (IRGC). With Annexes by Mike Roco and Emily Litten. Geneva: IRGC.

25. Ruzer LS (2008) Assessment of nanoparticles surface area by measuring of unattached fraction of radon progeny. J Nanopart Res 10:761-766. 
Citation: Ruzer LS (2011) Exposure and Dose: Health Effect Studies Associated with Nanometer Aerosols. J Nanomedic Nanotechnol 2:120. doi:10.4172/2157-7439.1000120

26. Ruzer LS (1964) The Method of Determining the Concentration of the Gas, Patent No. 234746 Bull Invent.

27. Dokukina VL, Ruzer LS (1976) All-Union Institute of Physico-Technical and Radiotechnical Measurements (VNIIFTRI), Moscow, 30 (60) (in Russian).

28. Ruzer LS, Sextro RG (1997) Assessment of Very Low Aerosol Concentrations by Measuring the Unattached Fraction of ${ }^{218} \mathrm{Po}$. Prot. Dosimetry 71: 135-140.

29. Butterweck G, Vezzu G, Schuler Ch, Muller R, Marsh JW, et al. (2001) In Vivo Measurement of Unattached Radon Progeny Deposited in the Human Respiratory Tract. Radiation Protection Dosimetry 94: 247-250.

30. Lassen L, Rau G (1960) The attachment of radioactive atoms on aerosols. Zeitschift f Physik 160: 504

31. Lassen L, Weicksel $H$ (1961) The attachment of radioactive atoms on aerosols in the radius range of $0.7-5 \mu \mathrm{m}$. Zeitschift $\mathrm{f}$ Physik 161: 339-345.
32. Croft JF, Perry KEG (1962) An experimental study of the behaviour and detection of hazardous radon releases in air. UK Atomic Energy Authority Reactor Group Report, AEEW - R 149.

33. Wilson WE, Stanek J, Pui DYH, Han HS (2004) Use of the Electrical Aeroso Detector as an Indicator for the Total Particle Surface Area Deposited in the Lung. Symposium on Air Quality Measurement Methods and Technology, Research Triangle Park, NC.

34. SCENIHR (2006) The appropriateness of existing methodologies to assess the potential risks associated with engineered and adventitious products of nanotechnologies. Health and Consumer Protection Directorate-General Scientific Committee on Emerging and Newly Identified Health Risks (SCENIHR). Brussels: European Commission.

35. NIOSH (2005) Strategic Plan for NIOSH Nanotechnology Research - Filling the Knowledge Gaps. Nanotechnology Research Program, National Institute for Occupational Safety and Health, Centers for Disease Control and Prevention. 